\title{
GmNAC81 Inversely Modulates Leaf Senescence and Drought Tolerance
}

\author{
Dalton O. Ferreira', Otto T. Fraga ${ }^{1,2}$, Maiana R. Pimenta ${ }^{3}$, Hanna D. N. Caetano, ${ }^{1,2}$ \\ João Paulo B. Machado4, Paola A. Carpinetti', Otávio J. B. Brustolinis, \\ lana P. S. Quadros ${ }^{1}$, Pedro A. B. Reis ${ }^{1,2}$ and Elizabeth P. B. Fontes ${ }^{1,2 *}$

\begin{abstract}
${ }^{1}$ National Institute of Science and Technology in Plant-Pest Interactions, Bioagro, Universidade Federal de Viçosa, Viçosa, Brazil, ${ }^{2}$ Department of Biochemistry and Molecular Biology/BIOAGRO, Universidade Federal de Viçosa, Viçosa, Brazil, ${ }^{3}$ Núcleo de Graduação de Agronomia, Universidade Federal de Sergipe, Nossa Senhora da Glória, Brazil, ${ }^{4}$ Agronomy Institute, Universidade Federal de Viçosa, Florestal, Brazil, ${ }^{5}$ National Laboratory for Scientific Computing (LNCC), Petrópolis,
\end{abstract} \\ Brazil
}

\section{OPEN ACCESS}

Edited by:

Zhong-Hua Chen, Western Sydney University, Australia

Reviewed by: Hui Song,

Qingdao Agricultural University, China Surendra Neupane University of Florida, United States

${ }^{*}$ Correspondence:

Elizabeth P. B. Fontes bbfontes@ufv.br

Specialty section: This article was submitted to Plant Genomics,

a section of the journal

Frontiers in Genetics

Received: 01 September 2020 Accepted: 26 October 2020 Published: 24 November 2020

Citation:

Ferreira DO, Fraga OT, Pimenta MR, Caetano HDN, Machado JPB, Carpinetti PA, Brustolini OJB, Quadros IPS, Reis PAB and Fontes EPB (2020) GmNAC81 Inversely Modulates Leaf Senescence and Drought Tolerance.

Front. Genet. 11:601876. doi: 10.3389/fgene.2020.601876
Glycine max NAC81 (GmNAC81) is a downstream effector of the DCD/NRP-mediated cell death signaling, which interacts with GmNAC30 to fully induce the caspase 1-like vacuolar processing enzyme (VPE) expression, the executioner of the cell death program. GmNAC81 has been previously shown to positively modulate leaf senescence via the NRP/GmNAC81/NPE signaling module. Here, we examined the transcriptome induced by GmNAC81 overexpression and leaf senescence and showed that GmNAC81 further modulates leaf senescence by regulating an extensive repertoire of functionally characterized senescence-associated genes (SAGs). Because the NRP/GmNAC81/NPE signaling circuit also relays stress-induced cell death signals, we examined the effect of GmNAC81 overexpression in drought responses. Enhanced GmNAC81 expression in the transgenic lines increased sensitivity to water deprivation. Under progressive drought, the GmNAC81-overexpressing lines displayed severe leaf wilting, a larger and faster decline in leaf $\Psi \mathrm{w}$, relative water content (RWC), photosynthesis rate, stomatal conductance, and transpiration rate, in addition to higher $\mathrm{Ci} / \mathrm{Ca}$ and lower Fm/Fv ratios compared to the BR16 control line. Collectively, these results indicate that the photosynthetic activity and apparatus were more affected by drought in the transgenic lines. Consistent with hypersensitivity to drought, chlorophyll loss, and lipid peroxidation were higher in the GmNAC81-overexpressing lines than in BR16 under dehydration. In addition to inducing VPE expression, GmNAC81 overexpression uncovered the regulation of typical drought-responsive genes. In particular, key regulators and effectors of ABA signaling were suppressed by GmNAC81 overexpression. These results suggest that GmNAC81 may negatively control drought tolerance not only via VPE activation but also via suppression of ABA signaling.

Keywords: GmNAC81, Glycine max, leaf senescence, drought tolerance, NAC transcription factors

\section{INTRODUCTION}

The NAC (an acronym for NAM, ATAF, and CUC) superfamily of plant-specific transcriptional factors (TF) is largely distributed in the plant kingdom and constitutes one of the largest families of plant TFs (Shao et al., 2015). The primary structural organization of NAC TFs includes a 150-aminoacid conserved $\mathrm{N}$-terminal DNA binding domain, designated the NAC domain, and a more variable C-terminal domain involved in transcriptional regulation (Nakashima et al., 2011). 
The NAC domain is divided into five subdomains (A-E). The $\mathrm{C}$ and $\mathrm{D}$ subdomains are highly conserved and are responsible for binding DNA. In contrast, the most divergent $\mathrm{B}$ and $\mathrm{E}$ subdomains may be responsible for the functional diversity of NAC genes, whereas the A subdomain may be involved in the formation of functional homo- or heterodimers or oligomers (Puranik et al., 2012). The D subdomain of some NACs may harbor a highly hydrophobic, negative regulatory domain (NRD), which inhibits NAC and TF partners (Puranik et al., 2012).

NAC proteins are mediators of responses to a range of developmental and environmental signals. Functionally characterized NACs display essential roles in flower development (Sablowski and Meyerowitz, 1998), apical meristem formation (Souer et al., 1996; Aida et al., 1997; Hibara et al., 2003), hormone signaling (Xie et al., 2000; Fujita et al., 2004), regulation of secondary cell wall biosynthesis (Mitsuda and Ohme-Takagi, 2008; Negi et al., 2017), regulation of leaf senescence (Kim et al., 2016), cell cycle control (Kim et al., 2006), lateral root development (Hao et al., 2011), pathogen infection responses (Collinge and Boller, 2001; Selth et al., 2005) and abiotic stress responses (Tran et al., 2004, 2010; Fang et al., 2008; Tweneboah and Oh, 2017; Chung et al., 2018). Therefore, the plant NAC superfamily relevance is incontestable, but functional studies of NAC genes in crop species are still limited.

In soybean, the superfamily encompasses 182 genes distributed evenly in the soybean chromosomes (Melo et al., 2018). Some NAC proteins are involved in both development and stress responses. Examples are GmNAC81 and GmNAC30, which form heterodimers to activate a cell death program via the upregulation of the caspase1-like vacuolar processing enzyme (VPE) expression (Hara-Nishimura et al., 2011; Mendes et al., 2013). Both GmNAC81 and GmNAC30 are coordinately regulated in response to several different stresses, cell death inducers, hormone signals, and developmentally programmed leaf senescence (Carvalho et al., 2014b; Pimenta et al., 2016).

GmNAC81 was first identified as a downstream component of the developmental cell death (DCD) domain-containing Asparagine (N)-rich protein, DCD/NRP-mediated cell death response (Faria et al., 2011; Reis et al., 2011; Reis and Fontes, 2012). This pathway has been described as a convergent response of osmotic and endoplasmic reticulum stress (Irsigler et al., 2007; Costa et al., 2008). The combination of both treatments promotes a synergistic effect in the DCD/NRPs expression mediated by the stress-induced transcriptional factor GmERD15 (Irsigler et al., 2007; Alves et al., 2011). The accumulation of DCD/NRP activates a signaling cascade leading to the upregulation of GmNAC81 and GmNAC30 (Mendes et al., 2013). In the nucleus, these TFs form a heterodimer that binds to a specific cis-regulatory element (TGTG[T/C/G]) on the VPE promoter to activate VPE expression, the executioner of a plant-specific programmed cell death (PCD) mediated by the collapse of the vacuole (Hara-Nishimura et al., 2011). In addition to VPE, the repertoire of GmNAC81 and GmNAC30 upregulated genes includes predominantly hydrolytic enzymes (carboxypeptidase, phosphatase, and glucanase) that may also be involved in plant PCD (Mendes et al., 2013). GmNAC81 has also been implicated as a positive modulator or developmentally programmed leaf senescence (Pimenta et al., 2016). Therefore, the NRP/GmNAC81:GmNAC30/VPE signaling module may function as a regulatory circuit that integrates developmentally programmed leaf senescence with stress-induced cell death. To gain insights into these linked responses' intricacy, we ectopically expressed GmNAC81 in soybean and analyzed the overlapping and specific features of these responses. We show here that in addition to promoting stress-induced cell death, GmNAC81 directly regulates drought-responsive genes and increases drought sensitivity.

\section{METHODS}

A detailed description of methods is provided in Supplementary Methods.

Briefly, GmNAC81-1 and GmNAC81-3 lines have been previously described (Pimenta et al., 2016). RNA sequencing and differential gene expression analyses were performed according to Melo et al. (2018). RNA-sequencing data were deposited in the Gene Expression Omnibus under accession number GSE159910 . Physiological parameter measurements were carried out as described (Pimenta et al., 2016), and water stress induction and monitoring were conducted according to Carvalho et al. (2014a).

\section{RESULTS}

\section{GmNAC81 Ectopic and Endogenous Expression Equalizes During the Onset of Leaf Senescence}

The transgenic lines GmNAC81-1 and GmNAC81-3 have been fully described previously (Pimenta et al., 2016). Here, we further complemented these previous studies by demonstrating first that despite accelerating leaf senescence (Figure 1A; Pimenta et al., 2016), GmNAC81 overexpression does not cause yield penalty.

The GmNAC81-overexpressing lines displayed accelerated leaf senescence (Figure 1A). At 90 days after germination (DAG), developmental stage R7, leaf yellowing was more intense in transgenic lines than in wild type (Figure 1A), which was associated with the higher accumulation of hydrogen peroxide in overexpressing lines, as judged by the intensity of DAB staining in senescent leaves (Supplementary Figure S1A). The precocious leaf senescence phenotype of the transgenic lines (Figure 1A) confirmed that the GmNAC81 transgene remained functional in the R5 generations of GmNAC81-1 and GmNAC81-3 lines, which were used for RNA-sequencing. Very importantly, the seed yield of GmNAC81-overexpressing lines was similar to that of the wild type (Figures 1B,C). The lower and greater pod numbers displayed by GmNAC81-1 and GmNAC81-3 lines, respectively, were compensated by differences in the weight of 1,000 seeds among genotypes in the growing seasons 2015/2016 and 2016/2017 (Supplementary Figures S1B,C). Therefore, seed weight/plant was similar in transgenic lines and wild type,

\footnotetext{
${ }^{1}$ https://www.ncbi.nlm.nih.gov/geo/query/acc.cgi?acc=GSE159910
} 
A

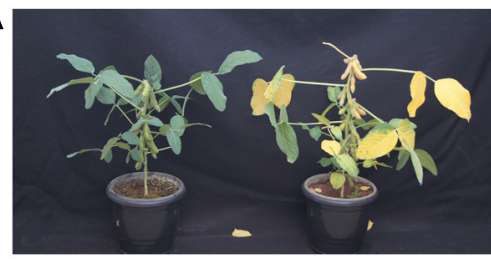

BR16

NAC81.1
B

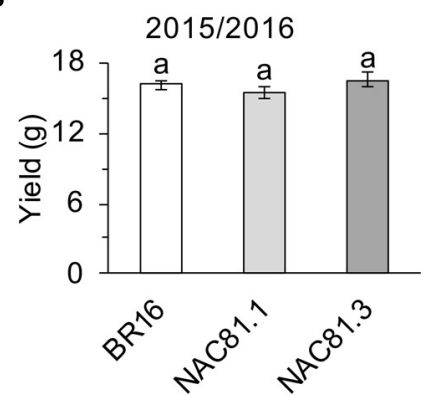

C

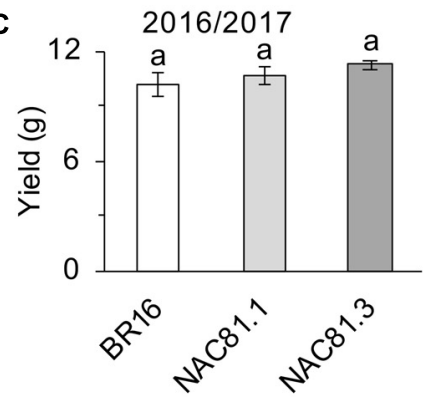

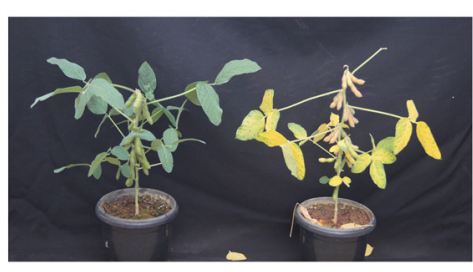

BR16

NAC81.3

D

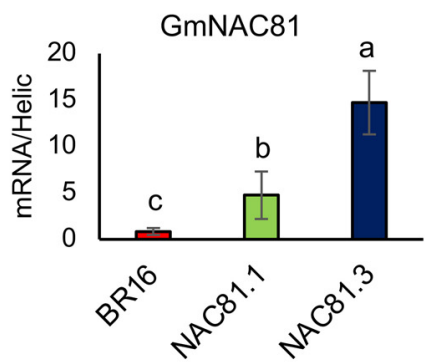

E

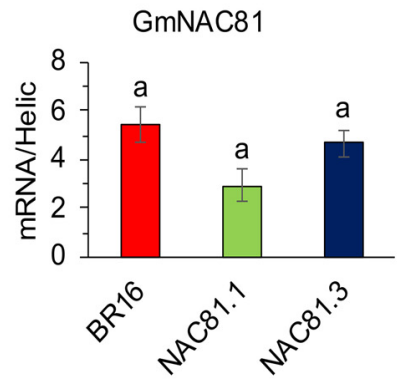

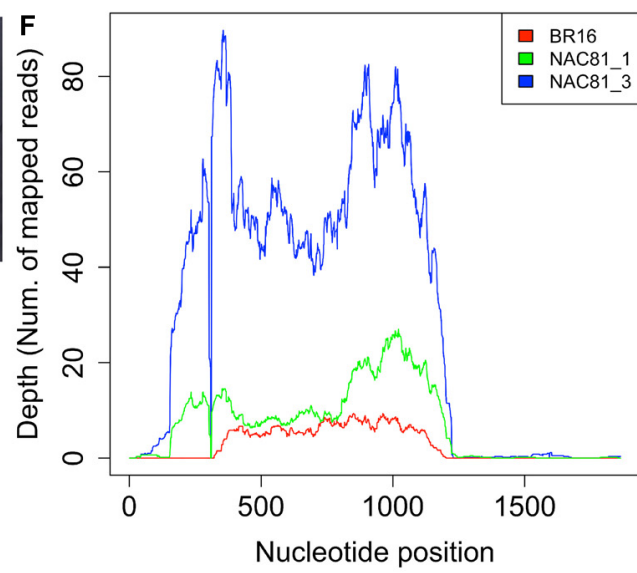

G

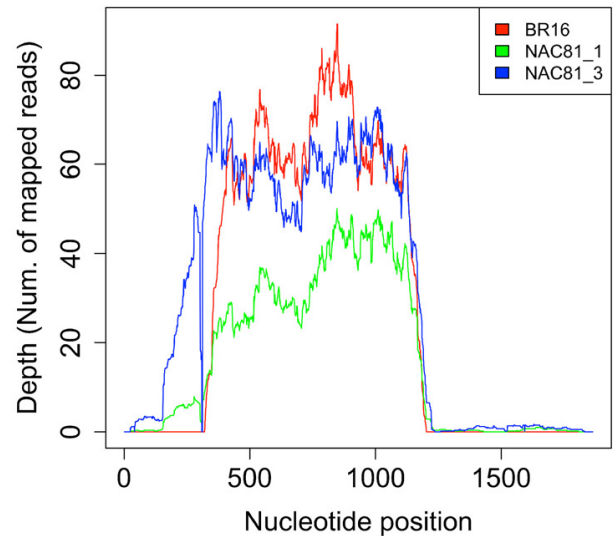

FIGURE 1 | GmNAC81 overexpression accelerates leaf senescence. (A) Phenotype of GmNAC81-overexpressing lines and the cultivar BR16 at 90 days after germination (DAG). (B) Mass of grains of BR16 and GmNAC81 lines in the first growing season (2015/2016) (mean \pm standard error). Means followed by different letters are significantly different by $t$-test $(p<0.05)$. (C) Mass of grains of BR16 and GmNAC81 lines in the second growing season (2016/2017) (mean \pm standard error). Means followed by different letters are significantly different by $t$-test $(p<0.05)$. (D) Normalized expression of GmNAC81 at 20 DAG. The accumulation of transcripts of GmNAC81 was determined by $2^{-\Delta \Delta \mathrm{Ct}}$ (mean \pm standard error). Means followed by different letters are significantly different by $t$-test $(p<0.05)$. (E) Normalized expression of GmNAC81 at 80 DAG. The accumulation of the GmNAC81 transcripts was determined by $2^{-\Delta} \Delta \mathrm{Ct}$ (mean \pm standard error). Means followed by different letters are significantly different by $t$-test $(p<0.05)$. (F) High throughput sequencing reads mapped to the GmNAC81 locus (Glyma.12G022700, Phytozome 12) at 20 DAG. (G) High throughput sequencing reads mapped to the GmNAC81 locus at 80 DAG.

indicating that GmNAC81 overexpression did not compromise yield (Figures 1B,C).

GmNAC81 is positively regulated during leaf senescence (Carvalho et al., 2014b; Pimenta et al., 2016). The RNA-seq data confirmed a strong upregulation of GmNAC81 during leaf senescence (Figures 1F,G). At 20 DAG (V3), GmNAC81 expression was significantly higher in GmNAC81-3 and slightly higher in GmNAC81-1 lines compared to BR16 (Figure 1D and Supplementary Figure S1F). In contrast, at 80 DAG (R6), endogenous GmNAC81 in the wild type was strongly induced, reaching a similar plateau as in the transgenic lines GmNAC81-3 and GmNAC81-1 (Figure 1G). These results were further confirmed by RT-qPCR, indicating that during the onset of leaf senescence, GmNAC81 ectopic expression in transgenic lines was similar to the endogenous expression in the wild-type, BR16 line (80 DAG; Figure 1E). Therefore, to delimitate the GmNAC81induced changes, we performed RNA-seq of GmNAC81-3 and BR16 leaves and compared the transcriptome changes in V3 leaves of GmNAC81_20DAG(V3) vs. BR16_20DAG(V3). Leaf senescence-induced changes were derived from both contrasts [BR16_80DAG(R6) vs. BR16_20DAG(V3)] and [GmNAC81_80DAG(R6) vs. GmNAC81_20DAG(V3)]. In the first contrast, we examined the effects of leaf senescence and GmNAC81 enhanced expression, and in the latter contrast, we analyzed leaf senescence-induced changes in GmNAC81 lines.

A global cluster analysis of the expressed sequences among BR16_20DAG leaves, BR16_80DAG leaves, GmNAC81_20DAG leaves, and GmNAC81_80DAG leaves revealed that the senescence-induced transcriptome of BR16 and GmNAC81 leaves at 80 DAG were most closely related; these samples clustered together with a high bootstrap probability and a high approximately unbiased $P$-value (Supplementary Figure S2A). These results confirmed that the effect of GmNAC81 overexpression is compensated by the strong induction of the 
endogenous gene during the onset of natural senescence (BR16 at $80 \mathrm{DAG}$ ), leading to similar responses during senescence.

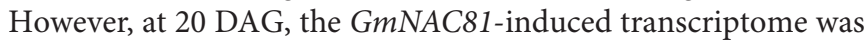
similar to the transcriptomes represented by the senescenceinduced transcriptome cluster and differed considerably from the BR16_20DAG transcriptome. These results indicated that the GmNAC81-mediated response and senescence-induced response shared similar mechanisms.

\section{GmNAC81 Overexpression Accelerates Leaf Senescence via the Induction of VPE and Senescence-Associated Genes}

GmNAC81 has been characterized as a downstream component of the DCD/NRP-mediated cell death response, induced by leaf senescence (Carvalho et al., 2014b; Pimenta et al., 2016). By using a corrected $p<0.05$ and log2-fold-change $>1$ or $\log 2$-fold-change $<1$ criteria for differentially regulated genes, our RNA-seq data confirmed that all previously described components of DCD/NRP-mediated cell death signaling (GmERD15, NRPs, GmNAC81, GmNAC30, and $\gamma$ VPE) were induced by leaf senescence (Supplementary Figure S3A). These results served as a validation criterion of our RNA-seq data. In the GmNAC81-overexpressing V3 leaves, the upstream components of GmNAC81 were not differentially expressed, except for ERD15, which was slightly downregulated [GmNAC81_20DAG(V3) vs. BR16_20DAG(V3)] (Supplementary Figure S3C). In contrast, two copies of VPE, which is a direct target of GmNAC81, were strongly induced, which may explain at least in part the accelerated senescence phenotype of the transgenic lines (Mendes et al., 2013; Carvalho et al., 2014b; Pimenta et al., 2016).

In the SNAC-A subfamily, GmNAC30 is most related to the Arabidopsis ATAF2 paralog and forms a cluster with GmNAC18 and GmNAC39 genes, which were induced by leaf senescence (Supplementary Figure S4). In contrast, the other four SNAC-A genes were repressed by leaf senescence (Supplementary Figure S3A); they are more divergent from GmNAC30 and clustered together with ATAF1 in a phylogenetic analysis (Supplementary Figure S4). GmNAC30 has been previously shown to interact with GmNAC81 to fully induce $\gamma$ VPE Glyma.14G092800 (Mendes et al., 2013). RNA-seq also demonstrated that the paralog $\gamma \mathrm{VPE}$ Glyma.17G230700 might be a direct target of GmNAC81 and GmNAC30. Accordingly, at the V3 stage (20 DAG), GmNAC81 overexpression in the transgenic leaves also induced both Glyma.14G092800 and Glyma.17G230700 VPEs, although to a lesser extent compared to their expression at 80 DAG (Supplementary Figures S3A-C). The $\gamma$ VPE paralog Glyma.04G049900 was also induced by leaf senescence (80 DAG) but not by overexpressing GmNAC81 in V3 leaves. These results are consistent with previous data showing that GmNAC81-regulated promoters also require GmNAC30 for full activation. In V3 leaves of GmNAC81-overexpressing lines, $G m N A C 30$ was not induced in parallel to provide a stoichiometric balance to the transcriptional activator complex.

To further validate the RNA-seq data, we examined the differential expression of other previously described
GmNAC81 target genes (Mendes et al., 2013) in the contrast GmNAC81_20DAG(V3) vs. BR16_20DAG(V3). Four upregulated and two downregulated genes by the complex GmNAC81:GmNAC30 were correctly induced and repressed by GmNAC81 overexpression in V3 leaves (Supplementary Figure S3D). These results further confirmed that the 35S:GmNAC81 transgene was functional.

To examine the transcriptional landscape of GmNAC81 during senescence, we compared the GmNAC81-induced transcriptome [GmNAC81_20DAG vs. BR16_20DAG] with the transcriptome induced by natural leaf senescence in BR16 [BR16_80DAG vs. BR16_20DAG] (Supplementary Figures S2B,C). Among the differentially expressed genes, $25.3 \%$ of upregulated genes and $42.7 \%$ of downregulated genes by GmNAC81 overexpression at 20 DAG were also differentially regulated during the onset of natural senescence at $80 \mathrm{DAG}$. To gain further insights into the cellular processes affected by GmNAC81, we performed functional enrichment analyses of the differentially expressed genes identified in the contrast [GmNAC81_20DAG vs. BR16_20DAG]. Significantly enriched GO terms (with $p<0.05$ ) were identified in all three categories, Biological Process, Molecular Function, or Cellular Component ontology. Consistent with the function of GmNAC81 in senescence, a subset of DEGs was significantly over-represented under senescence-related GO terms (Figure 2A and Supplementary Tables S1, S2).

Under the leaf senescence-enriched class, we selected a subset of induced and repressed genes for confirmatory analysis of gene expression by RT-qPCR (Supplementary Table S3). The upregulated senescence-associated genes included NTR1.5 (Nitrate Transporter 1.5), KIN10, a catalytic subunit of SnRK1 (sucrose non-fermenting 1-related kinase 1 homolog 10) (Kim et al., 2016), MLO3 (seven-transmembrane domain mildew resistance locus $\mathrm{O} 3$ protein (Piffanelli et al., 2002), and the wounding-induced JMT (Jasmonate O-methyltransferase) (Turner et al., 2002). NTR1.5 is a transmembrane protein responsible for nitrate transport, strongly induced by leaf senescence (Diaz et al., 2005, 2008; Fan et al., 2009). The selected KIN10 interacts directly and phosphorylates ETHYLENE INSENSITIVE 3, destabilizing an essential TF involved in ethylene signaling and senescence (Kim et al., 2016). Therefore, KIN10 negatively regulates ethylene signaling and hence may explain at least in part the massive downregulation of ethyleneresponsive genes as a result of GmNAC81 overexpression (Supplementary Table S2). In particular, $M L O 3$ was strongly induced by GmNAC81 overexpression at 20 DAG and natural leaf senescence. MLO3 belongs to the plant-specific Mlo family of proteins with seven transmembrane segments and modulates defense- and environmentally induced cell death (Devoto et al., 1999; Piffanelli et al., 2002). The wounding-induced JMT promotes JA methylation to the activated form MeJA (Turner et al., 2002) and accelerates leaf senescence (Chena et al., 2017).

Among the downregulated genes, we selected KIT1 (Kunitz trypsin inhibitor 1), induced by wounding and involved in programmed cell death (PCD) (Li et al., 2008, 2012) and SPI (serine protease inhibitor), a regulator of PCD (Clemente et al., 2019). The results of RT-qPCR confirmed the RNA-seq data. At 
20 DAG, NRT1.5, MLO3, JMT, and KIN10 genes were induced by enhanced expression of GmNAC81, whereas the KIT1 and SPI genes were repressed (Figure 2B). The level of induction and repression of these representative genes correlated with transgene expression levels in the independently transformed lines GmNAC81-1 and GmNAC81-3 (Figure 1D). Furthermore, the promoters of the selected genes harbor variable numbers of GmNAC81 cis-regulatory elements [TGTG(TGC)]. The positions of GmNAC81 binding sites on the respective promoter and probability against random genomic sequences are shown in Supplementary Table S4. There are five repetitions of the GmNAC81 DNA binding site on the $5^{\prime}$ flanking sequences of NTR1.5 with a probability of 0.004 against random genomic sequence, indicating that NTR1.5 is a potential target of

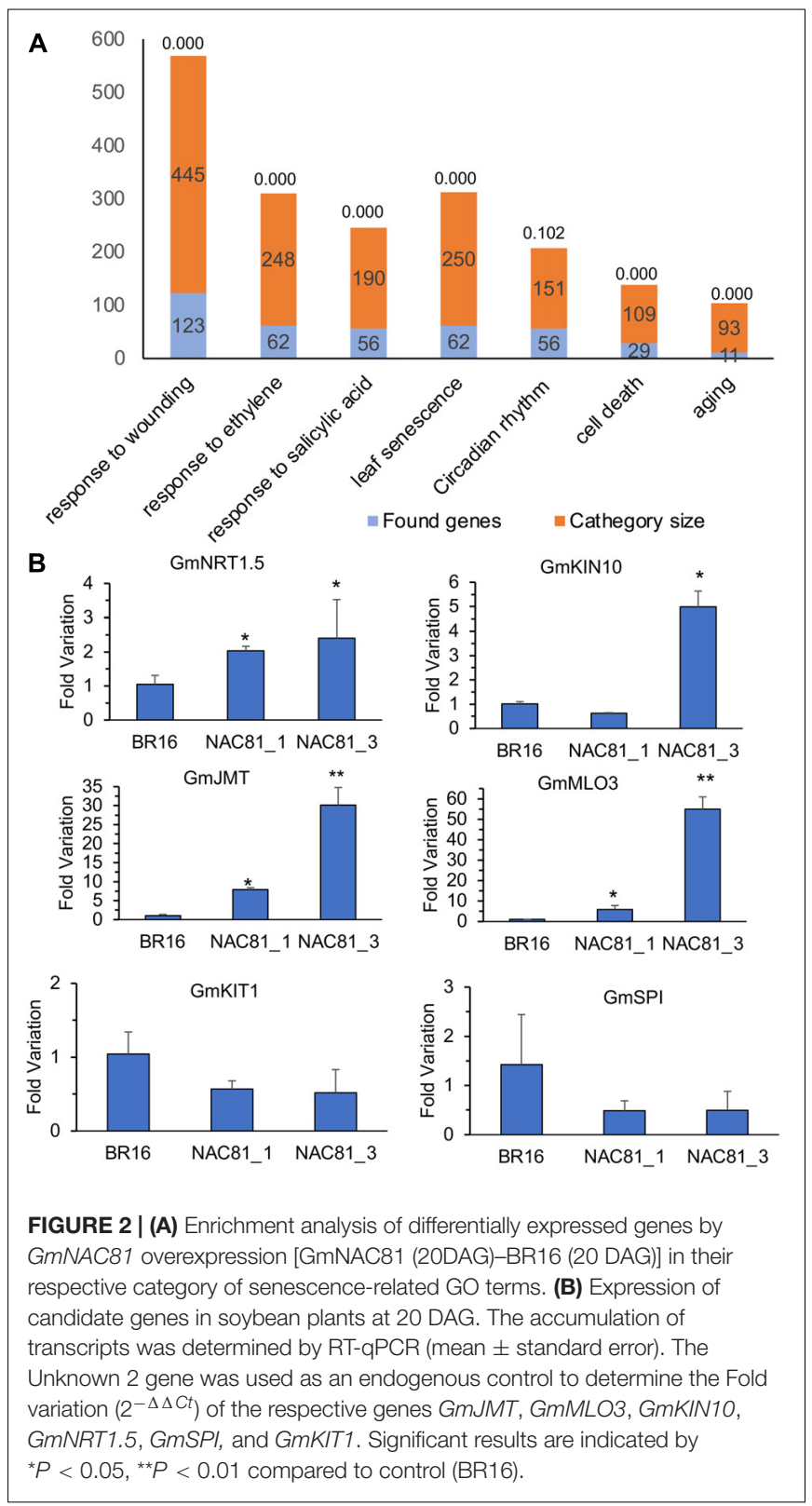

GmNAC81. KIN10 promoter harbors three repetitions, MLO3 and KIT1 promoters, two repetitions. MLO3 and JMT promoters contain one single GmNAC81 binding site. Collectively, these data suggest that the selected senescence-related genes are potential targets of GmNAC81.

\section{GmNAC81 Overexpression Increases Sensitivity to Drought Tolerance}

The negative modulation of the DCD/NRP-mediated cell death signaling enhances tolerance to drought (Carvalho et al., 2014a; Reis et al., 2016). Ectopic expression of the molecular chaperone binding protein (BiP) inhibits the expression and activity of NRPs, GmNAC81, and VPE and confer drought tolerance (Valente et al., 2009; Reis et al., 2011; Carvalho et al., 2014a). These previous results raised the hypothesis that mechanisms underlying drought tolerance may be associated with leaf longevity. To further examine this hypothesis, the GmNAC81-overexpressing lines, GmNAC81-1 and GmNAC813 at the developmental stage V3, were exposed to a progressive reduction of irrigation for 20 days. Under these dehydration conditions, BR16 and the transgenic lines GmNAC81-1 and GmNAC81-3 maintained similar water potential until the 12th day of water deprivation (Figure 3A). After 12 days, however, the leaf water potential decreased to a different extent in BR16 and the transgenic lines, resulting in accelerated leaf wilting in transgenic lines (Figure 3B). After 20 days under progressive dehydration, the leaf water potential of the stressed GmNAC81-3 line dropped to a maximum stress of $-2.0 \mathrm{MPa}$, whereas the stressed GmNAC81-1 needed an additional day to reach this maximum stress. BR16 leaf water potential reached $-2.0 \mathrm{MPa}$ after 23 days of exposition to the restricted water regime. Under progressive dehydration, a clear difference in RWC between BR16 and transgenic (GmNAC81-1 and GmNAC81-3) leaves was observed at 17 days and persisted until 19 days after the treatment (Figure 3C). Collectively, these results indicate that, under progressive drought, GmNAC81 overexpression enhances sensitivity to dehydration.

Under normal growth conditions, the photosynthetic rate (A) of transgenic lines was superior to that of BR16 (Figure 3D), which may explain the accelerated development of GmNAC81-1 and GmNAC81-3 lines (Pimenta et al., 2016). Accordingly, GmNAC81 overexpression induced the expression of photosynthetic apparatus components (Supplementary Figure S5). We also showed that the precocious senescence of transgenic lines did not cause yield loss, which may be explained by the higher photosynthetic rate of the GmNAC81-1 and GmNAC81-3 lines. However, during progressive water deficit, the photosynthetic rate decline was more pronounced in the GmNAC81-1 and GmNAC81-3 lines compared to BR16, dropping to zero at 17 days in the transgenic lines but not until 20 days in BR16 (Figure 3D). The declines in stomatal conductance (gs) and transpiration rate (E) paralleled that of the photosynthetic rate (Supplementary Figures S6A,B). Therefore, under drought, the alterations in photosynthesis and transpiration rates may have been 

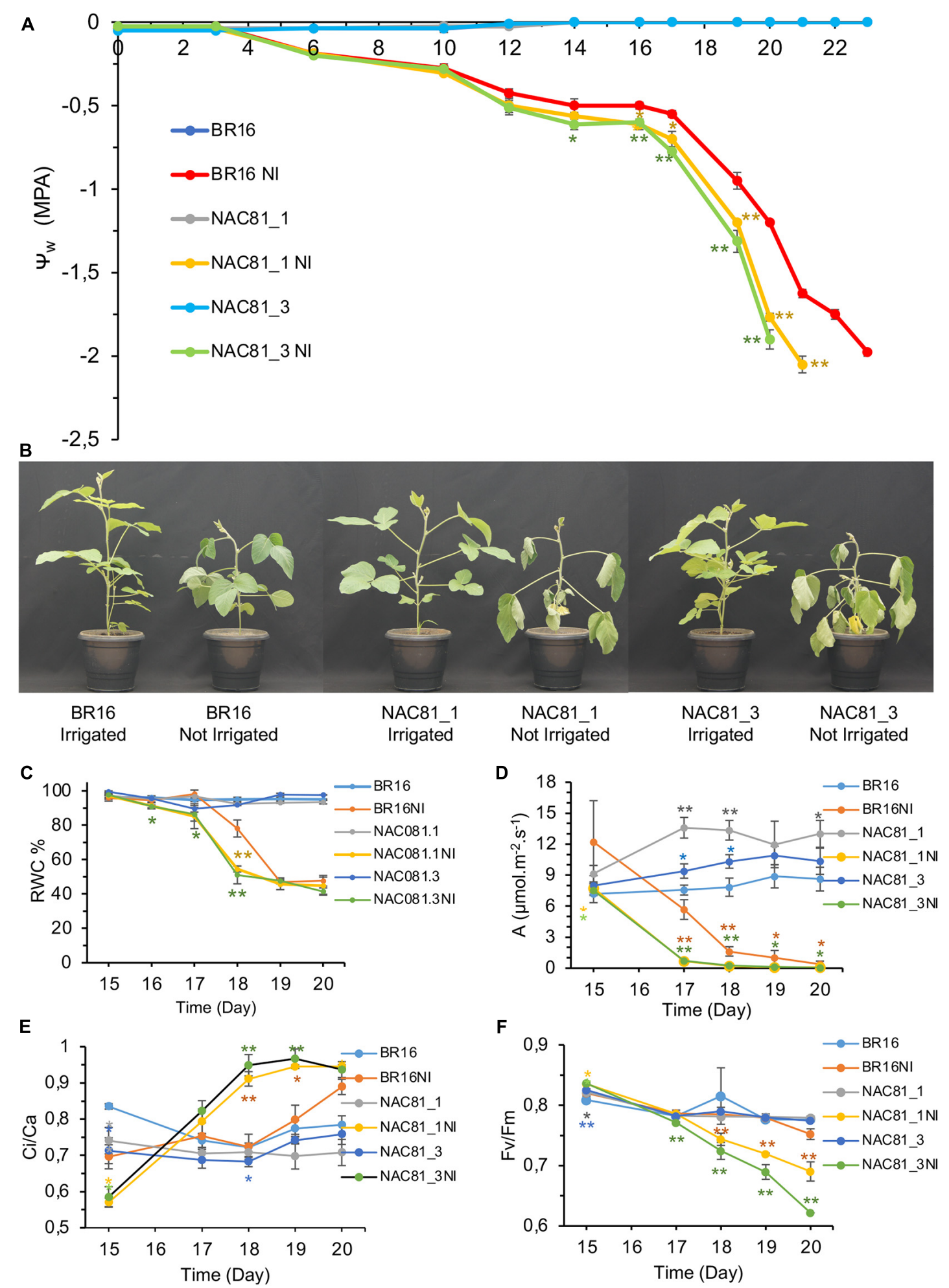

FIGURE 3 | Soybeans plants in V3 stage submitted to a progressive water deficit regime. Drought stress was induced by a gradual reduction of the daily normal water supply. (A) Leaf water potential was measured with a Scholander pump over time. Numbers are days after the exposition to the water deficit regime.

(B) Phenotype of soybean plants when the water potential reached -1.0 MPa in unirrigated BR16. (C) Relative water content of time. (D) Physiological parameters of gas exchange along the gradual drought stress Photosynthesis (D,E) Ci/Ca ratio. (F) Fluorescence physiological parameter, Fv/Fm ratio, throughout the gradual drought stress. The bars represent the standard error $(n=4)$. Significant results are indicated by ${ }^{\star} P<0.05$, ${ }^{\star \star} P<0.01$, compared to BR16 control lines. NI indicated not irrigated. 
a direct result of stomatal closure, which occurred more rapidly in the transgenic lines; therefore, the mechanism for GmNAC81-mediated enhanced sensitivity to dehydration may not be associated with a failure in drought-induced stomatal closure.

Despite continuous stomatal closure during the progressive water deficit, the $\mathrm{Ci} / \mathrm{Ca}$ ratio was higher in the GmNAC81overexpressing lines than in the wild-type, indicating less $\mathrm{CO}_{2}$ fixation in the transgenic lines (Figure 3E). Only at 20 days of stress, the $\mathrm{Ci} / \mathrm{Ca}$ ratio of $\mathrm{BR} 16$ was similar to that of the transgenic lines. During progressive water deprivation, the carboxylation efficiency (A/Ci ratio) was reduced more drastically in GmNAC81-overexpressing lines than in BR16 (Supplementary Figure S6C). Collectively, these results suggest that the more accentuated reduction in the $\mathrm{CO}_{2}$ assimilation rate in the GmNAC081-overexpressing lines during leaf dehydration might have been caused by biochemical damage of the photosynthetic apparatus.

The instantaneous water-use efficiency (A/E) progressively declined during the water deficit in both BR16 and transgenic lines, although to a different extent (Supplementary Figure S6D). A/E declined more rapidly and accentually in GmNAC81-overexpressing lines than in BR16, which maintained a superior A/E during all stress periods. The intrinsic water-use efficiency (A/gs) displayed the same trend as $\mathrm{A} / \mathrm{E}$
(Supplementary Figure S6E). The photosynthetic activity of the soybean lines was also evaluated by measuring the maximum photochemical efficiency of PSII (Fv/Fm) (Figure 3F) and the electron transport rate (ETR) (Supplementary Figure S6F). Fv/Fm and ETR progressively declined during the water deficit regime, although significantly more in stressed GmNAC81-1 and GmNAC81-3 leaves than in stressed BR16 leaves. These results reflect a higher degree of drought-induced stress in the transgenic lines.

Consistent with the enhanced drought susceptibility phenotype of GmNAC81-3, photosynthetic pigments, including chlorophyll $a$, chlorophyll $b$, and carotenoids, were more affected by water deficit in the GmNAC81-3 leaves than in GmNAC81-1 followed by BR16 (Figures 4A-C). Likewise, the extent of lipid peroxidation measured by the level of TBA-reactive compounds was higher in stressed GmNAC81-3 leaves than in stressed GmNAC81-1 and BR16 leaves (Figure 4D). These results correlated with the expression level of the GmNAC81, leaf gas-exchange data, and chlorophyll-fluorescence parameters. Furthermore, they confirmed that GmNAC81 is a negative modulator of drought tolerance.

As GmNAC81 is a downstream component of the multiple stress-induced DCD/NRP-mediated cell death response, the primary hypothesis was that GmNAC81 promotes susceptibility to drought due to its capacity to accelerate cell death induced by
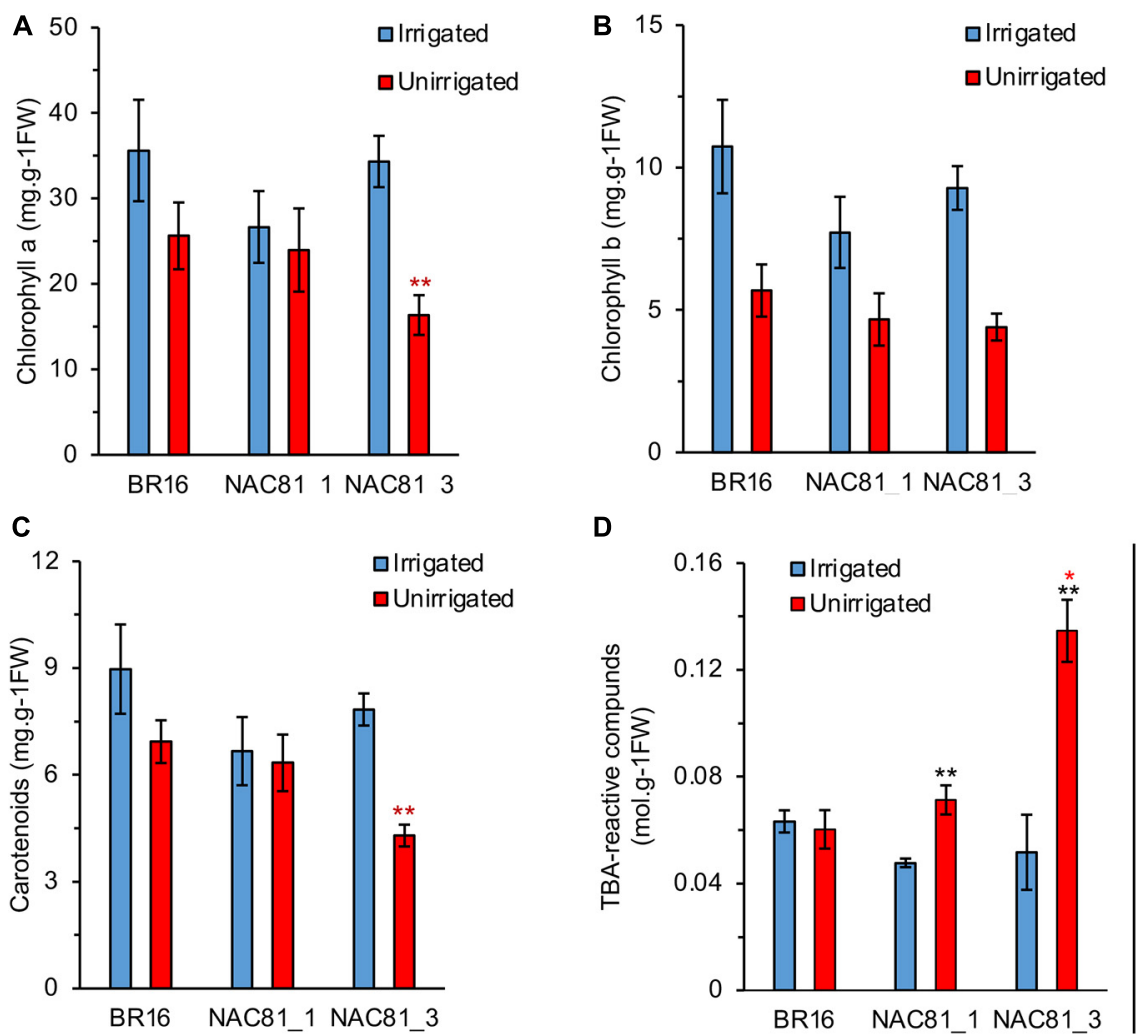

FIGURE 4 | Pigment contents in drought-stressed soybeans plants. (A) Chlorophyll a. (B) Chlorophyll b. (C) Carotenoids. (D) Thiobarbituric acid-reactive compounds. The bars represent the standard error $(n=4)$. Significant results are indicated by ${ }^{\star} P<0.05$, ${ }^{\star \star} P<0.01$. The colored asterisks indicate the significantly different GmNAC81 line compared to BR16. The black asterisks indicate significant difference between treatments of same genotype. 
multiple stresses. We showed that GmNAC81 targets senescenceassociated genes and accelerates senescence. These senescenceassociated GmNAC81-regulated genes were common to the contrast GmNAC81_20DAG vs. BR16_20DAG and BR1_80DAG vs. BR16_20DAG. To examine whether GmNAC81 would also be directly involved in drought tolerance by targeting typical drought-responsive genes, we analyzed differentially expressed genes exclusively present in the contrast GmNAC81_20DAG vs. BR16_20DAG (Supplementary Table S5). Under normal growth conditions, the GmNAC81 overexpression predominantly promoted the downregulation of drought-responsive genes. Among GmNAC81-repressed drought-responsive genes, we found major players in typical drought response. These include PDR12 (AtPDR12)/ABCG40 (pleiotropic drug resistance transporter), which encodes a plasma membrane transporter that promotes ABA uptake (Kang et al., 2010), genes encoding aquaporins involved in water transport regulation in plant cells, such as PIP2A plasma membrane intrinsic protein $2 \mathrm{~A}$ and TMP-C, plasma membrane intrinsic protein 1. Furthermore, GmNAC81 downregulated CBF4, a member of the DREB A1 subfamily of TFs belonging to the ERF/AP2 family (Haake et al., 2002; Guttikonda et al., 2014). These results suggest that the drought sensitivity phenotype of GmNAC81-overexpressing lines may be associated not only with the GmNAC81 positive regulation of cell death and leaf senescence but also with the capacity of GmNAC81 to repress essential genes involved in the assembly of a drought-tolerant response.

\section{DISCUSSION}

GmNAC81 is a downstream component of the multiple stressand natural leaf senescence-induced DCD/NRP-mediated cell death signaling. Here, we further confirmed the essential role of GmNAC81 as a positive regulator of developmentally programmed leaf senescence and uncovered a GmNAC81 negative role in drought response. Indeed, GmNAC81 overexpression in the early vegetative stage accelerated leaf senescence and increased sensitivity to drought. Our data confirmed that the GmNAC81/VPE regulatory circuit accounts at least in part for the enhanced drought sensitivity of plants via induced PCD but also showed that GmNAC81 might act directly to modulate negatively typical drought response.

The genome-wide identification of the transcriptional activity of GmNAC81 revealed that the underlying mechanism for GmNAC81-mediated senescence is not only associated with the caspase 1-like VPE function, but also with upregulation of functionally characterized senescence-associated genes. Among the differentially expressed genes mediated by GmNAC81 overexpression in the early vegetative stage (20 DAG), $25.3 \%$ upregulated and $42.7 \%$ downregulated genes were common to the transcriptional landscape of natural leaf senescence. In addition to previously described GmNAC81 targets, we found an overrepresentation of differentially expressed genes under biological categories related to cell death, leaf senescence, JA, and SA signaling. GmNAC81-regulated expression of six genes, JMT, MLO3, NRT1.5, KIN10, KIT1, and SPI, which function in leaf senescence, was further validated by RT-qPCR. These senescence-associated genes harbor GmNAC81 DNA binding sites [TGTG(TGC)] on their promoter, substantiating the argument that they may be direct targets for GmNAC81 control.

GmNAC81 also functions as a transcriptional repressor (Mendes et al., 2013), contributing to its positive function in leaf senescence. Approximately $43 \%$ of downregulated genes by GmNAC81 overexpression at 20 DAG were also downregulated by natural leaf senescence. PCD not only requires the activation of proteases, as pro-apoptotic factors but also the coordinate suppression of protease inhibitors to modulate the progress of cell death. As protease inhibitors and negative modulators of cell death during plant development and senescence, KIT1 (Li et al., 2008) and STI (Clemente et al., 2019) were highly repressed by the GmNAC81 overexpression at 20 DAG. Therefore, GmNAC81 not only directly targets VPE, as previously characterized (Mendes et al., 2013; Carvalho et al., 2014b), but also induces functionally described SAGs and suppresses inhibitors of leaf senescence, as potential direct targets.

In addition to accelerating leaf senescence, we provided several lines of evidence that GmNAC81 enhances sensitivity to water dehydration. First, under progressive water deficit, the GmNAC81-overexpressing lines, GmNAC81-1 and GmNAC813, maintained a lower leaf $\Psi_{\mathrm{W}}$ and RWC than the control, BR16. Second, progressive drought damaged the photosynthetic apparatus of the transgenic lines more rapidly than the control BR16. Accordingly, the photosynthesis rate, transpiration rate, and stomatal conductance declines were more pronounced in the transgenic lines, which displayed higher $\mathrm{Ci} / \mathrm{Ca}$ and lower Fv/Fm ratios than BR16. Third, drought-induced pigment loss, including chlorophyll and carotenoids, and lipid peroxidation were higher in transgenic lines than in BR16, confirming that drought-induced cell death was enhanced in transgenic lines, which was consistent with the GmNAC81-induced transcriptome. These results also confirmed the hypothesis that modulation of the NRP-mediated cell death signaling is linked to drought response modulation. While ectopic expression of a negative modulator of this cell death signaling confers drought tolerance (Valente et al., 2009; Reis et al., 2011; Carvalho et al., 2014a), we showed here that overexpression of the positive regulator GmNAC81 enhanced drought sensitivity.

Our results also indicated that cell death and other typical drought tolerance mechanisms might operate under the negative control of GmNAC81. Accordingly, drought-responsive genes were overrepresented in the set of genes specifically down-regulated by $G m N A C 81$ overexpression but not by leaf senescence. This set of genes uncouples GmNAC81-mediated leaf senescence from GmNAC81-mediated drought response. Among the GmNAC81 specifically down-regulated genes, we found classical TFs involved in drought response, including CBF4, a member of the DREB A-1 subfamily of the ERF/AP2 family (Haake et al., 2002; Guttikonda et al., 2014). CBF4 is a critical regulator of gene expression during drought adaptation and is induced by drought in an abscisic acid (ABA)-dependent manner. The ATP-binding cassette $(\mathrm{ABC})$ transporter pleiotropic drug resistance transporter PDR12 (AtPDR12)/ABCG40, a plasma membrane ABA uptake transporter, was also repressed 
in GmNAC81-overexpressing V3 leaves but not during the onset of leaf senescence (R6 leaves). Inactivation of PDR12 in tobacco cells impairs ABA uptake and delays ABA-responsive genes (Kang et al., 2010). The Zinc finger protein ZAT10 was found to be specifically downregulated by GmNAC81 overexpression in V3 leaves. ZAT10 functions as both transcriptional activator and repressor involved in abiotic stress responses (Mittler et al., 2006); it controls the expression of the stress-responsive genes DREB1A and LTI78. Likewise, the regulators of ABA signaling, AtHD2C and OST1, were specifically downregulated in GmNAC81-overexpressing V3 leaves. As a functional droughtresponsive gene, $A t H D 2 C$ overexpression in Arabidopsis affects the expression of several ABA-responsive genes and enhances tolerance to salt and drought stresses (Sridha and $\mathrm{Wu}, 2006$ ). OST1 is an ABA-activated protein kinase, which regulates stomatal closure by ABA but does not affect stomatal regulation by light and $\mathrm{CO} 2$, implicating OST1 specifically in ABA signaling (Mustilli et al., 2002). Collectively, these data suggest that GmNAC81 directly modulates drought tolerance by controlling critical regulators of ABA signaling. These results implicate the GmNAC81 regulatory function as a convergent link between stress-induced cell death signals and drought responses.

\section{DATA AVAILABILITY STATEMENT}

The datasets generated for this study can be found in the online repositories. The names of the repository/repositories and accession number(s) can be found in the article/ Supplementary Material.

\section{AUTHOR CONTRIBUTIONS}

DF performed most of the experiments, analyzed the data, and wrote the first draft of the manuscript. OF performed the RT-qPCR. MP designed and assisted the drought stress experiments. $\mathrm{HC}$ assisted the drought stress experiments. JM and PC performed the RT-qPCR and carried-out RNA-seq. $\mathrm{OB}$ performed the bioinformatic and RNA-seq analysis. IQ performed the RT-qPCR of transgenic lines. PR designed the experiments. EF designed the experiments, analyzed the data, and wrote the manuscript. All authors contributed to the article and approved the submitted version.

\section{REFERENCES}

Aida, M., Ishida, T., Fukaki, H., Fujisawa, H., and Tasaka, M. (1997). Genes involved in organ separation in Arabidopsis: an analysis of the cup-shaped cotyledon mutant. Plant Cell 9, 841-857. doi: 10.1105/tpc.9. 6.841

Alves, M. S., Reis, P. A. B., Dadalto, S. P., Faria, J. A. Q. A., Fontes, E. P. B., and Fietto, L. G. (2011). A novel transcription factor, ERD15 (Early Responsive to Dehydration 15), connects endoplasmic reticulum stress with an osmotic stressinduced cell death signal. J. Biol. Chem. 286, 20020-20030. doi: 10.1074/jbc. M111.233494

Carvalho, H. H., Brustolini, O. J. B., Pimenta, M. R., Mendes, G. C., Gouveia, B. C., Silva, P. A., et al. (2014a). The molecular chaperone binding protein BiP

\section{FUNDING}

We are grateful to the Brazilian funding agencies CAPES finance code 001, CNPq, FAPEMIG, and the National Institute of Science and Technology in Plant-Pest interactions for supporting this study.

\section{SUPPLEMENTARY MATERIAL}

The Supplementary Material for this article can be found online at: https://www.frontiersin.org/articles/10.3389/fgene. 2020.601876/full\#supplementary-material

Supplementary Figure 1 | DAB staining of leaves at 80 DG and yield components of GmNAC81-1, GmNAC81-3, and BR16 plants.

Supplementary Figure 2 | GmNAC81-mediated variation in the expression of senescence-related genes.

Supplementary Figure $\mathbf{3}$ | Leaf senescence induces the expression of DCD/NRP-mediated programmed cell death signaling pathway.

Supplementary Figure 4 | Phylogenetic tree of the SNAC-A subfamily (ATAF) of the NAC family.

Supplementary Figure 5 | GmNAC81-mediated differential expression of the photosynthetic apparatus components [GmNAC81 (20DAG) × BR16 (20DAG)].

Supplementary Figure 6 | Physiological parameters of gas exchange during progressive drought stress.

Supplementary Table 1 | Enrichment analysis of genes differentially expressed [35S:GmNAC81 (20DAG)-BR16 (20 DAG)] related to senescence and the respective GO category.

Supplementary Table 2 | Functional GO-based categorization of senescence related genes, differentially expressed in the contrast GmNAC81 (20 DAG)-BR16 (20 DAG).

Supplementary Table 3 | Examples of genes differentially expressed shared by [35S:GmNAC81 (20DAG)-BR16 (20DAG)] vs. [35S:GmNAC81 (80 DAG)-BR16 (80DAG)].

Supplementary Table 4 | Candidate targets of GmNAC81 harboring the binding site TGTG[T/G/C] in 5' flanking sequences.

Supplementary Table 5 | Genes differentially expressed involved in drought stress between 35S: GmNAC81 and BR16 plants at 20 DAG.

Supplementary Table 6 | List of oligonucleotides used in the qRT-PCR assays. Supplementary Methods | Detailed description of Materials and Methods.

prevents leaf dehydration-induced cellular homeostasis disruption. PLoS One 9:e86661. doi: 10.1371/journal.pone.0086661

Carvalho, H. H., Silva, P. A., Mendes, G. C., Brustolini, O. J., Pimenta, M. R., Gouveia, B. C., et al. (2014b). The endoplasmic reticulum binding protein BiP displays dual function in modulating cell death events. Plant Physiol. 164, 654-670. doi: 10.1104/pp.113.23192

Chena, Y., Wanga, Y., Huanga, J., Zhenga, C., Caib, C., Wangb, Q., et al. (2017). Salt and methyl jasmonate aggravate growth inhibition and senescence in Arabidopsis seedlings via the JA signaling pathway. Plant Sci. 261, 1-9. doi: 10.1016/j.plantsci.2017.05.005

Chung, P. J., Jung, H., Choi, Y. D., and Kim, J. K. (2018). Genome-wide analyses of direct target genes of four rice NAC-domain transcription factors involved in drought tolerance. BMC Genomics 19:40. doi: 10.1186/s12864-017-4367-1 
Clemente, M., Corigliano, M. G., Pariani, S. A., Sánchez-López, E. F., Sander, V. A., and Ramos-Duarte, V. A. (2019). Plant serine protease inhibitors: biotechnology application in agriculture and molecular farming. Int. J. Mol. Sci. 20:1345. doi: 10.3390/ijms20061345

Collinge, M., and Boller, T. (2001). Differential induction of two potato genes, Stprx 2 and StNAC, in response to infection by Phytophthora infestans and to wounding. Plant Mol. Biol. 46, 521-529. doi: 10.1023/A:1010639225091

Costa, M. D., Reis, P. A., Valente, M. A., Irsigler, A. S., Carvalho, C. M., Loureiro, M. E., et al. (2008). A new branch of endoplasmic reticulum stress signaling and the osmotic signal converge on plant-specific asparagine-rich proteins to promote cell death. J. Biol. Chem. 283, 20209-20219. doi: 10.1074/jbc. M802654200

Devoto, A., Piffanelli, P., Nilsson, I., Wallin, E., Panstruga, R., von Heijne, G., et al. (1999). Topology, subcellular localization, and sequence diversity of the Mlo family in plants. J. Biol. Chem. 274, 34993-35004. doi: 10.1074/jbc.274.49.34993

Diaz, C., Lemaître, T., Christ, A., Azzopardi, M., Kato, Y., Sato, F., et al. (2008). Nitrogen recycling and remobilization are differentially controlled by leaf senescence and development stage in Arabidopsis under low nitrogen nutrition. Plant Physiol. 147, 1437-1449. doi: 10.1104/pp.108.119040

Diaz, C., Purdy, S., Christ, A., Morot-Gaudry, J.-F., Wingler, A., and MasclauxDaubresse, C. (2005). Characterization of markers to determine the extent and variability of leaf senescence in Arabidopsis. A metabolic profiling approach. Plant Physiol. 138, 898-908. doi: 10.1104/pp.105.060764

Fan, S.-C., Lin, C.-S., Hsu, P.-K., Lin, S.-H., and Tsay, Y.-F. (2009). The Arabidopsis nitrate transporter NRT1. 7, expressed in phloem, is responsible for source-tosink remobilization of nitrate. Plant Cell 21, 2750-2761. doi: 10.1105/tpc.109. 067603

Fang, Y., You, J., Xie, K., Xie, W., and Xiong, L. (2008). Systematic sequence analysis and identification of tissue-specific or stress-responsive genes of NAC transcription factor family in rice. Mol. Genet. Genomics 280, 547-563. doi: 10.1007/s00438-008-0386-6

Faria, J. A. Q. A., Reis, P. A. B., Reis, M. T. B., Rosado, G. L., Pinheiro, G. L., Mendes, G. C., et al. (2011). The NAC domain-containing protein, GmNAC6, is a downstream component of the ER stress- and osmotic stress-induced NRPmediated cell-death signaling pathway. BMC Plant Biol. 11:129. doi: 10.1186/ 1471-2229-11-129

Fujita, M., Fujita, Y., Maruyama, K., Seki, M., Hiratsu, K., Ohme-Takagi, M., et al. (2004). A dehydration-induced NAC protein, RD26, is involved in a novel ABAdependent stress-signaling pathway. Plant J. 39, 863-876. doi: 10.1111/j.1365313X.2004.02171.x

Guttikonda, S. K., Valliyodan, B., Neelakandan, A. K., Tran, L. S., Kumar, R., Quach, T. N., et al. (2014). Overexpression of AtDREB1D transcription factor improves drought tolerance in soybean. Mol. Biol. Rep. 41, 7995-8008. doi: 10.1007/s11033-014-3695-3

Hake, V., Cook, D., Riechmann, J. L., Pineda, O., Thomashow, M. F., and Zhang, J. Z. (2002). Transcription factor CBF4 is a regulator of drought adaptation in Arabidopsis. Plant Physiol. 130, 639-648. doi: 10.1104/pp.006478

Hao, Y. J., Wei, W., Song, Q. X., Chen, H. W., Zhang, Y. Q., Wang, F., et al. (2011). Soybean NAC transcription factors promote abiotic stress tolerance and lateral root formation in transgenic plants. Plant J. 68, 302-313. doi: 10.1111/j.1365313X.2011.04687.x

Hara-Nishimura, I., Hatsugai, N., Nakaune, S., Kuroyanagi, M., and Nishimura, M. (2011). Vacuolar processing enzyme: an executor of plant cell death. Curr. Opin. Plant Biol. 8, 404-408. doi: 10.1016/j.pbi.2005.05.016

Hibara, K., Takada, S., and Tasaka, M. (2003). CUC1 gene activates the expression of SAM-related genes to induce adventitious shoot formation. Plant J. 36, 687-696. doi: 10.1046/j.1365-313x.2003.01911.x

Irsigler, A. S. T., Costa, M. D. L., Zhang, P., Reis, P. A. B., Dewey, R. E., Boston, R. S., et al. (2007). Expression profiling on soybean leaves reveals integration of ER- and osmotic-stress pathways. BMC Genomics 8:431. doi: 10.1186/14712164-8-431

Kang, J., Hwang, J. U., Lee, M., Kim, Y. Y., Assmann, S. M., Martinoia, E., et al. (2010). PDR-type ABC transporter mediates cellular uptake of the phytohormone abscisic acid. Proc. Natl. Acad. Sci. U.S.A. 107, 2355-2360. doi: 10.1073/pnas.0909222107

Kim, H. J., Nam, H. G., and Lim, P. O. (2016). Regulatory network of NAC transcription factors in leaf senescence. Curr. Opin. Plant Biol. 33, 48-56. doi: 10.1016/j.pbi.2016.06.002
Kim, Y. S., Kim, S. G., Park, J. E., Park, H. Y., Lim, M. H., Chua, N. H., et al. (2006). A membrane-bound NAC transcription factor regulates cell division in Arabidopsis. Plant Cell. 18, 3132-3144. doi: 10.1105/tpc.106.043018

Li, J., Brader, G., and Palva, E. T. (2008). Kunitz trypsin inhibitor: an antagonist of cell death triggered by phytopathogens and fumonisin B1 in Arabidopsis. Mol. Plant 1, 482-495. doi: 10.1093/mp/ssn013

Li, Z., Peng, J., Wen, X., and Guo, H. (2012). Gene network analysis and functional studies of senescence-associated genes reveal novel regulators of Arabidopsis leaf senescence. J. Integr. Plant Biol. 54, 526-539. doi: 10.1111/j.1744-7909.2012. 01136.x

Melo, B. P., Fraga, O. T., Silva, J. C. F., Ferreira, D. O., Brustolini, O. J. B., Carpinetti, P. A., et al. (2018). Revisiting the soybean GmNAC superfamily. Front. Plant Sci. 9:1864. doi: 10.3389/fpls.2018.01864

Mendes, G. C., Reis, P. A., Calil, I. P., Carvalho, H. H., Aragão, F. J., and Fontes, E. P. (2013). GmNAC30 and GmNAC81 integrate the endoplasmic reticulum stress- and osmotic stress-induced cell death responses through a vacuolar processing enzyme. Proc. Natl. Acad. Sci. U.S.A. 110, 19627-19632. doi: 10. 1073/pnas.1311729110

Mitsuda, N., and Ohme-Takagi, M. (2008). NAC transcription factors NST1 and NST3 regulate pod shattering in a partially redundant manner by promoting secondary wall formation after the establishment of tissue identity. Plant J. 56, 768-778. doi: 10.1111/j.1365-313X.2008.03633.x

Mittler, R., Kim, Y., Song, L., Coutu, J., Coutu, A., Ciftci-Yilmaz, S., et al. (2006). Gain- and loss-of-function mutations in Zat10 enhance the tolerance of plants to abiotic stress. FEBS Lett. 580, 6537-6542. doi: 10.1016/j.febslet.2006.11.002

Mustilli, A.-C., Merlot, S., Vavasseur, A., Fenzi, F., and Giraudat, J. (2002). Arabidopsis OST1 protein kinase mediates the regulation of stomatal aperture by abscisic acid and acts upstream of reactive oxygen species production. Plant Cell 14, 3089-3099. doi: 10.1105/tpc.007906

Nakashima, K., Takasaki, H., Mizoi, J., Shinozaki, K., and Yamaguchi-Shinozaki, K. (2011). NAC transcription factors in plant abiotic stress responses. Biochim. Biophys. Acta 1819, 97-103. doi: 10.1016/j.bbagrm.2011.10.005

Negi, S., Tak, H., and Ganapathi, T. R. (2017). Native vascular related NAC transcription factors are efficient regulator of multiple classes of secondary wall associated genes in banana. Plant Sci. 265, 70-86. doi: 10.1016/j.plantsci.2017. 09.018

Piffanelli, P., Zhou, F., Casais, C., Orme, J., Jarosch, B., Schaffrath, U., et al. (2002). The barley MLO modulator of defense and cell death is responsive to biotic and abiotic stress stimuli. Plant Physiol. 129, 1076-1085. doi: 10.1104/pp.010954

Pimenta, M. R., Silva, P. A., Mendes, G. C., Alves, J. R., Caetano, H. D., Machado, J. P., et al. (2016). The stress-induced soybean NAC transcription factor GmNAC81 plays a positive role in developmentally programmed leaf senescence. Plant Cell Physiol. 57, 1098-1114. doi: 10.1093/pcp/pcw059

Puranik, S., Sahu, P. P., Srivastava, P. S., and Prasad, M. (2012). NAC proteins: regulation and role in stress tolerance. Trends Plant Sci. 17, 369-381. doi: 10.1016/j.tplants.2012.02.004

Reis, P. A. B., Carpinetti, P. A., Freitas, P. P. J., Santos, E. G. D., Camargos, L. F., Oliveira, I. H. T., et al. (2016). Functional and regulatory conservation of the soybean ER stress-induced DCD/NRP mediated cell death signaling in plants. BMC Plant Biol. 16:156. doi: 10.1186/s12870-016-0843-Z

Reis, P. A. B., and Fontes, E. P. B. (2012). N-rich protein (NRP)-mediated cell death signaling: a new branch of the ER stress response with implications for plant biotechnology. Plant Signal. Behav. 7, 628-632. doi: 10.4161/psb.20111

Reis, P. A. B., Rosado, G. L., Silva, L. A., Oliveira, L. C., Oliveira, L. B., Costa, M. D., et al. (2011). The binding protein BiP attenuates stress induced cell death in soybean via modulation of the N-rich protein-mediated signaling pathway. Plant Physiol. 157, 1853-1865. doi: 10.1104/pp.111.179697

Sablowski, R. W., and Meyerowitz, E. M. (1998). A homolog of NO APICAL MERISTEM is an immediate target of the floral homeotic genes APETALA3/PISTILLATA. Cell 92, 93-103. doi: 10.1016/s0092-8674(00)8 0902-2

Selth, L. A., Dogra, S. C., Rasheed, M. S., Healy, H., Randles, J. W., and Rezaian, M. A. (2005). A NAC domain protein interacts with tomato leaf curl virus replication accessory protein and enhances viral replication. Plant Cell 17, 311-325. doi: 10.1105/tpc.104.027235

Shao, H., Wang, H., and Tang, X. (2015). NAC transcription factors in plant multiple abiotic stress responses: progress and prospects. Front. Plant. Sci. 6:902. doi: 10.3389/fpls.2015.00902 
Souer, E., van Houwelingen, A., Kloos, D., Mol, J., and Koes, R. (1996). The no apical meristem gene of Petunia is required for pattern formation in embryos and flowers and is expressed at meristem and primordia boundaries. Cell 85, 159-170. doi: 10.1016/s0092-8674(00)81093-4

Sridha, S., and Wu, K. (2006). Identification of AtHD2C as a novel regulator of abscisic acid responses in Arabidopsis. Plant J. 46, 124-133. doi: 10.1111/j.1365313X.2006.02678.x

Tran, L. S. P., Nakashima, K., Sakuma, Y., Simpson, S. D., Fujita, Y., Maruyama, K., et al. (2004). Isolation and functional analysis of Arabidopsis stress-inducible NAC transcription factors that bind to a drought-responsive cis-element in the early responsive to dehydration stress 1 Promoter. Plant Cell 16, 2481-2498. doi: $10.1105 /$ tpc.104.022699

Tran, L. S. P., Nishiyama, R., Yamaguchi-Shinozaki, K., and Shinozaki, K. (2010). Potential utilization of NAC transcription factors to enhance abiotic stress tolerance in plants by biotechnological approach. GM Crops 1, 32-39. doi: 10.4161/gmcr.1.1.10569

Turner, J. G., Ellis, C., and Devoto, A. (2002). The jasmonate signal pathway. Plant Cell 14, 153-164. doi: 10.1105/tpc.000679

Tweneboah, S., and Oh, S.-K. (2017). Biological roles of NAC transcription factors in the regulation of biotic and abiotic stress responses in solanaceous crops. J. Plant Biotechnol. 44, 1-11. doi: 10.5010/JPB.2017.44.1.001
Valente, M. A. S., Faria, J. A. Q. A., Soares-Ramos, J. R., Reis, P. A. B., Pinheiro, G. L., Piovesan, N. D., et al. (2009). The ER luminal binding protein (BiP) mediates an increase in drought tolerance in soybean and delays drought induced leaf senescence in soybean and tobacco. J. Exp. Bot. 60, 533-546. doi: $10.1093 /$ jxb/ern296

Xie, Q., Frugis, G., Colgan, D., and Chua, N. H. (2000). Arabidopsis NAC1 transduces auxin signal downstream of TIR1 to promote lateral root development. Genes Dev. 14, 3024-3036. doi: 10.1101/gad.852200

Conflict of Interest: The authors declare that the research was conducted in the absence of any commercial or financial relationships that could be construed as a potential conflict of interest.

Copyright (C) 2020 Ferreira, Fraga, Pimenta, Caetano, Machado, Carpinetti, Brustolini, Quadros, Reis and Fontes. This is an open-access article distributed under the terms of the Creative Commons Attribution License (CC BY). The use, distribution or reproduction in other forums is permitted, provided the original author(s) and the copyright owner(s) are credited and that the original publication in this journal is cited, in accordance with accepted academic practice. No use, distribution or reproduction is permitted which does not comply with these terms. 